\title{
Morphology lies: a case-in-point with a new non-biting midge species from Oriental China (Diptera, Chironomidae)
}

\author{
Chao Song', Xinhua Wang², Wenjun $\mathrm{Bu}^{2}$, Xin $\mathrm{Qi}^{1}$ \\ I College of Life Sciences, Taizhou University, Taizhou, Zhejiang 318000, China 2 College of Life Sciences, \\ Nankai University, Tianjin, 300071, China \\ Corresponding author: Xin Qi (qixin0612@tzc.edu.cn)
}

Academic editor: F.L. da Silva | Received 22 August 2019 | Accepted 17 December 2019 | Published 5 February 2020

http://zoobank.org/2D411E81-F5CB-4906-9845-BA82E04F5616

Citation: Song C, Wang X, Bu W, Qi X (2020) Morphology lies: a case-in-point with a new non-biting midge species from Oriental China (Diptera, Chironomidae). ZooKeys 909: 67-77. https://doi.org/10.3897/zookeys.909.39347

\begin{abstract}
Morphological traits are generally indicative of specific taxa, and particularly function as keys in taxonomy and species delimitation. In this study, a non-biting midge species with an Einfeldia-like superior volsella makes it hard to accurately determined based on its morphological characteristics. Molecular genes of two ribosomal genes and three protein-encoding genes were compiled to construct a related genera phylogeny and to address the taxonomic issues. Phylogenetic inference clearly supports the undetermined species as belonging to Kiefferulus. Therefore, a new species classified in the genus Kiefferulus is described and figured as an adult male from Oriental China. The species could be easily distinguished from other species in having an Einfeldia-like superior volsella and a triangular tergite IX.
\end{abstract}

\section{Keywords}

COI, CAD, Kiefferulus, morphology, phylogeny, PGD, taxonomy

\section{Introduction}

For hundreds of years, taxonomists have been mainly focused on morphological characteristics for classification, taxonomy, and species identification. The most essential part of traditional taxonomy is based on similarities and differences to create systematics. Linnaeus (1753) simplified and standardized the nomenclature into the binomial system

Copyright Chao Song et al. This is an open access article distributed under the terms of the Creative Commons Attribution License (CC BY 4.0), which permits unrestricted use, distribution, and reproduction in any medium, provided the original author and source are credited. 
of genus and species. However, the system created is mainly based on visible characteristics by taxonomists' own professional experience, which is unstable and difficult to test. Discoveries and naming of new organisms aim to seek natural groupings with different proxies, such as morphology, genes, ecology, and behavior (Holstein and Luebert 2017). Nevertheless, classification of insects has been based on morphological characteristics to a great extent, which means that one species is deemed to be related with another based on shared characteristics of the same origin (synapomorphies).

With the burgeoning of molecular technology, there have been heated debates among scientists on whether the traditional system should be retained (Garnett and Christidis 2017; Thomson et al. 2018). Some think that the classification of complex organisms is in chaos and hampers species conservation, while others argue that taxonomy is necessary for global species conservation. After more than 250 years of the predominance of comparative morphology in species discovery, advanced methods and technology, especially molecular data, are rapidly expanding the realm of taxonomy (Padial et al. 2010). In addition, molecular information of certain species is increasingly registered or recorded and made available via several global initiatives, such as National Center for Biotechnology Information (NCBI), Barcode of Life Data System (BOLD), and different local barcode libraries (Ratnasingham and Hebert 2007). However, integrative taxonomy requires both detailed morphology description and molecular inference, which is time-consuming. Recently, regarding new species description, it is preferable to provide both morphology and COI barcodes, but COI-based phylogeny inference is unstable and not always convincing. Consequently, with the acceleration of new species descriptions, there would be much likely the peril of erroneous species hypotheses and unstable names (Padial et al. 2010)

Chironomidae is a large family of diverse flies and commonly called non-biting midges. It is the most widely distributed of all aquatic insect families occurring in all zoogeographical region of the world, including Antarctica (Cranston et al. 1989). It also shows adaptions to different extreme niches, surviving at elevations of 5,600 m of Himalaya Mountains (Kohshima 1984) and at more than 1,000 m depth in Lake Baikal (Linnevich 1971).

Kiefferulus was described by Goetghebuer (1922) to accommodate Tanytarsus tedipediformis from Belgium (Chaudhuri and Ghosh 1986). However, it was later recognized as a subgenus of Pentapedilum Kiefer by Edwards (1929) and of Chironomus Meigen by Townes (1945), after which Hamilton et al. (1969) restored its generic status. The male Kiefferulus is easily recognized by its characteristic hypopygium, such as the broadly sickle-shaped superior volsella with numerous long setae on the inner margin and long microtrichia reaching the distal part, and the distal inferior volsella being strongly expanded (Cranston et al. 1989).

Herein, we used sequences from two ribosomal genes (18S and $28 \mathrm{~S}$ ribosomal DNA), three protein-encoding genes [cytochrome oxidase I (COI), CPSase region of carbamol-phosphate synthase-aspartate transcarbamolylase-dihydroorotase (CAD), and phosphogluconate dehydrogenase (PGD)] to explore the undetermined chirono- 
mid species' systemic position. Through phylogenetic relationships, it is recognized as a new species of Kiefferulus based on molecular phylogeny analysis. We also discuss whether morphological traits can be independently used to define species within nonbiting midges. Finally, Kiefferulus trigonum sp. nov. is presented and described.

\section{Materials and methods}

\section{Taxon sampling}

The morphological nomenclature follows Sæther (1980). The examined specimens were mounted on slides following the procedure by Sæther (1969). Measurements are given as ranges followed by the mean when there are four or more specimens examined. All types are deposited in College of Life Science, Nankai University.

Digital photographs were captured with a Leica DFC420 camera using a Leica DM6000 B compound microscope and under the application of the software Leica Suite at the NTNU university Museum, NTNU (Trondheim, Norway). Photograph postprocessing were done in Adobe photoshop and Illustrator (Adobe Inc., California, USA).

\section{DNA extraction, PCR amplification, sequencing, and alignment}

Tissues for total genome DNA extraction were removed from the thorax, heads of adult, and abdomen of larvae. The extraction procedure followed the Qiagen DNeasy Blood and Tissue kit except for elusion buffer ranging from 100-150 $\mu \mathrm{l}$ according to different body sizes. After extraction, the exoskeletons were cleared and mounted to corresponding voucher numbers. We amplified two ribosomal genes (18S and 28S) and four protein coding gene segments including fragments of one mitochondrial gene (COI-3P), two sections of the CPSase region of carbamoylphosphate synthase-aspartate transcarbamoylase-dihydroorotase (CAD1 and CAD4), and phosphogluconate dehydrogenase (PGD). Besides, universal primers LCO1490 and HCO2198 were used for the standard COI barcode sequences.

Polymerase Chain Reaction (PCR) amplifications were done in a $25 \mu$ l volume including $12.5 \mu \mathrm{l} 2 \times$ Es Taq MasterMix (CoWin Biotech Co., Beijing, China), $0.625 \mu \mathrm{l}$ of each primer, $2 \mu \mathrm{l}$ of template DNA and $9.25 \mu \mathrm{l}$ deionized $\mathrm{H}_{2} \mathrm{O}$, or $2.5 \mu \mathrm{l} 10 \times$ Takara ExTaq buffer (CL), $2 \mu \mathrm{l} 2.5$ mM dNTP mix, $2 \mu \mathrm{l} 25 \mathrm{mM} \mathrm{MgCl} 2,0.2 \mu \mathrm{l}$ Takara Ex Taq HS, $1 \mu \mathrm{l} 10 \mu \mathrm{M}$ of each primer, $2 \mu \mathrm{l}$ template DNA and $14.3 \mu \mathrm{ddH}_{2} \mathrm{O}$. PCR was performed on a PowerCylcer Gradient SL (Biometra Gmbh, Göttingen, Germany). For the mitochondrial gene, the program was set as follows: an initial denaturation step of $95^{\circ} \mathrm{C}$ for $5 \mathrm{~min}$, then followed by 34 cycles of $94^{\circ} \mathrm{C}$ for $30 \mathrm{~s}, 51^{\circ} \mathrm{C}$ for $30 \mathrm{~s}, 72{ }^{\circ} \mathrm{C}$ for $1 \mathrm{~min}$ and final extension at $72^{\circ} \mathrm{C}$ for $3 \mathrm{~min}$. The program of ribosomal genes and nuclear protein coding genes were referred to Cranston et al. (2012), alternatively for the protein coding genes that a touchdown program: initial denaturation step of $98^{\circ} \mathrm{C}$ 
for $10 \mathrm{~s}$, then $94^{\circ} \mathrm{C}$ for $1 \mathrm{~min}$ followed by five cycles of $94^{\circ} \mathrm{C}$ for $30 \mathrm{~s}, 52^{\circ} \mathrm{C}$ for $30 \mathrm{~s}$, $72^{\circ} \mathrm{C}$ for $2 \mathrm{~min}$ and 7 cycles of $94^{\circ} \mathrm{C}$ for $30 \mathrm{~s}, 51^{\circ} \mathrm{C}$ for $1 \mathrm{~min}, 72^{\circ} \mathrm{C}$ for $2 \mathrm{~min}$ and 37 cycles of $94^{\circ} \mathrm{C}$ for $30 \mathrm{~s}, 45^{\circ} \mathrm{C}$ for $20 \mathrm{~s}, 72^{\circ} \mathrm{C}$ for $2 \mathrm{~min} 30 \mathrm{~s}$ and one final extension at $72{ }^{\circ} \mathrm{C}$ for $3 \mathrm{~min}$. PCR product were confirmed on a $1 \%$ agarose gel and sequenced in both directions with ABI 3730 or ABI 3730XL capillary sequencers at Beijing Genomics Institute Co., Ltd, Beijing, China.

DNA sequences were edited and assembled with BioEdit 7.0.1 (Hall 1999). We applied the appropriate IUPAC code when editing the raw sequences in case of ambiguous bases but use "?" instead of the ambiguity symbol "N" in the matrix. Sequence matrix of protein coding genes were aligned by their amino acid sequences using Muscle (Edgar 2004) in MEGA7 (Kumar et al. 2016). Introns in CAD and PGD were recognized and deleted according to refence sequences and "GT-AG" rule before analysis. For two ribosomal genes sequences were aligned by muscle and then removed the poorly aligned positions using Glocks online server (http://phylogeny.lirmm.fr/ phylo_cgi/one_task.cgi?task_type=gblocks) (Castresana 2000; Dereeper et al. 2008).

\section{Phylogenetic analysis}

Maximum likelihood (ML) trees were constructed in raxml-GUI v1.5b2 (Silvestro and Michalak 2012), with 1000 bootstrap replicates in a rapid bootstrap analysis, using GTR+G+I substitution model with partitions. Bayesian inference analysis (BI) was performed in two parallel runs in MrBayes (Nylander et al. 2004), consisting each of four chains of six million generations with a sampling frequency 1000 generation for one tree and burin of 25\%. Partitions were in PartionFinder using greedy search and selected according to aicc (Lanfear et al. 2012). Result was as follows: $T R N+I+G$ for 18S, COI3_2; GTR+I+G for COI3p_p1, 28S; GTR+I+G for CAD4_P1, CAD1_P1, PGD_P1; GTR+I+G for CAD1_P2, CAD4_P2, PGD_P2; GTR+I+G for CAD4_P3, PGD_P3; HKY+G for COI3p_P3; HKY+I+G for CAD1_P3. The convergence was checked in Tracer v1.7 (Rambaut et al. 2014) and terminated when ESS were superior to 200 with the initial $25 \%$ trees as burn in.

\section{Results}

The initial sequences of genes are CAD1 909bp, CAD4 846 bp, PGD 747 bp, 18S 933 bp, COI3P 826 bp, and 28S 743 bp (DOI: dx.doi.org/10.5883/DS-KIFFER). To reduce the effects of missing data, we trimmed the beginning and end of the protein coding genes and delete highly variable regions of $18 \mathrm{~S}$ and $28 \mathrm{~S}$ and finally concatenated to 4335 bp (CAD1 828 bp, CAD4 760 bp, PGD 747 bp, COI3P 662 bp, 18S 852 bp, $28 \mathrm{~S} 455 \mathrm{bp}$ ) (SI). Both ML and BI inference show the same topology (Fig. 1) and agree on the simple phylogenetic scenario: the odd species conflict with the morphotype genus of Einfeldia but are clearly supported as species of Kiefferulus. 


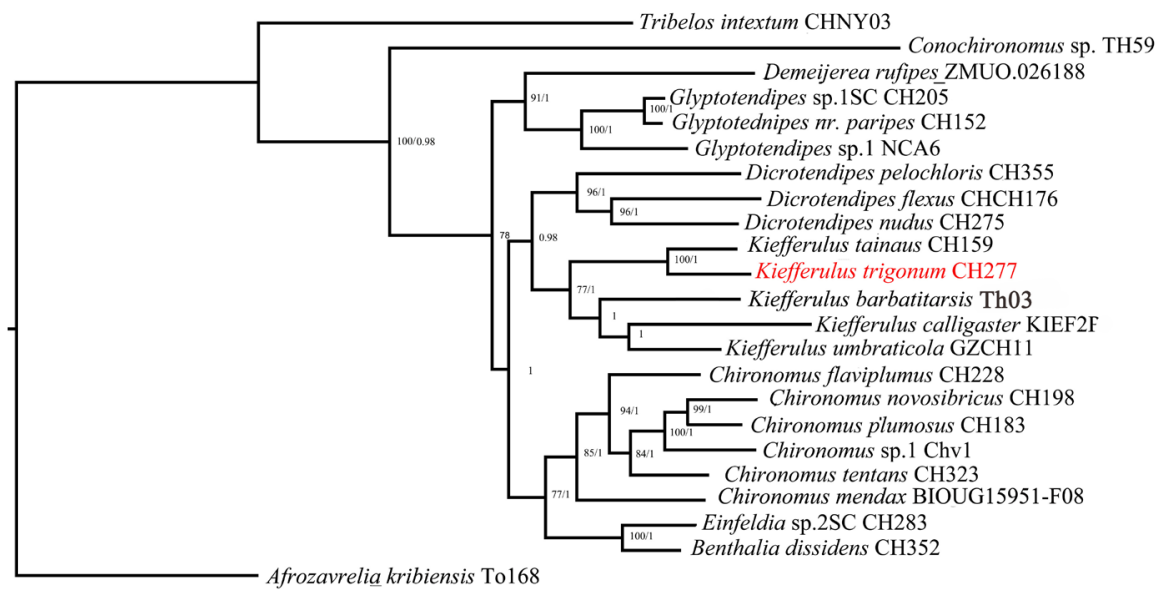

Figure I. Bayes Inference tree (BI) tree based on the concatenated DNA dataset (18S, 28S, COI3P, $\mathrm{CAD} 1, \mathrm{CAD} 4, \mathrm{PGD})$ of Kiefferulus and its related genera; Afrozavrelia was used as the outgroup. Numbers on branches refer to ML bootstrap values over $75 \%$ and posterior probabilities over 0.95 .

The new species was not identified using morphological taxonomic keys for adult Chironomidae (Cranston et al. 1989). The superior volsella with a large hairy base and a digitiform bare projection was recognized as an important and diagnostic definition of Einfeldia sensu lato, which makes it a pre-identification as an Einfeldia sp. Nevertheless, the typical superior volsella is not exclusive, and also occurs in Benthalia, Chironomus (including its subgenera Chironomus and Lobochironomus), Conochironomus, Glyptotendipes, and Tribelos. From morphological parsimony analysis, these genera sharing similar superior volsella are not closely related (Andersen et al. 2017). Molecular phylogeny of the related genera in this study, and in Cranston et al. (2012) show that Conochironomus and Glyptotendipes are not closely related to Einfeldia. Consequently, generic complexes or species groups with Einfeldia-like superior volsella are not genetically monophyletic clades (Fig. 1). Such cases of convergent characters are likely to causes serious problems in phylogenetic analysis, and lead to misplacement of species or genus. The hypothesis of generic diagnosis has raised great confusion within adult taxonomy. The case is not unique in Chironomidae: the marine species Dicrotendipes sinicus Qi \& Lin was suggested as a new genus within the subfamily Chironominae. However, the analysis of genetic data revealed that the marine species nested within the genus Dicrotendipes (Qi et al. 2019).

To clearly illustrate the species' systemic position, it was included in the molecular phylogeny of related genera. Surprisingly, the morphologically identified species fall within the clade of Kiefferulus (Fig 1). Obviously, morphology-based identification conflicts with the molecular phylogeny. Considering the morphological homoplasy and phenotypic changes, we clarified the Einfeldia-like species within the genus 
Kiefferulus. While the Einfeldia-type superior volsella is unique in Kiefferulus, the new species is named as Kiefferulus trigonum sp. nov.

When defining a species new to science, almost no taxonomists would test its systemic position, which would be time-consuming and costly. Hierarchical classifications based on appropriate morphological characters provide a main backbone of the life tree, while molecular data provide corroboration, resolution, and support (Scotland et al. 2003). Genera defined and recognized by clear morphological characters as in Chironomidae, such as Wiederholm (1989) for adults, Andersen et al. (2013) for larvae, and Wiederholm (1986) for pupae have not been tested with a full molecular phylogeny. Morphology alone was not enough to make a correct placement especially for some hyper diverse genera or monotypic genera and the traditional taxonomy needs revisions according to molecular phylogeny.

\section{Taxonomy}

Kiefferulus trigonum sp. nov. http://zoobank.org/E880611F-E713-4FCC-9F82-1696F96008A5 Figs 2, 3, BIN: ADG9680

Type material. Holotype (BDN No. 041685) 10, China, Fujian Province: Longyan City, Shanghang County, Buyun Town, Qiushan, 25.03N, 116.24E, 6.V.1993, Wang $\mathrm{XH}$, light trap. Paratypes: $1 \widehat{O}^{\lambda}$ same as holotype; $3 \hat{\jmath}$, Fujian Province: Sanming City,

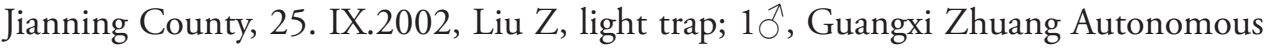
Region: Nanning City, 9. V.1986, Wang XH; 20 , Guizhou Province: Libo County,

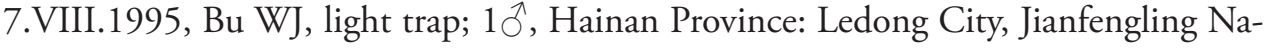
tional Forest Park, Song C, light trap.

Etymology. From Latin, trigonum means triangle, referring to the triangular tergite IX.

Diagnostic characters. The male adults could be obviously distinguished from other Kiefferulus species by the triangular IX tergite, superior volsella with projection and basal part of inferior volsella wider than distal part.

Description. Male imago $(N=9)$. Total length 4.78-5.90, $5.30 \mathrm{~mm}$; wing length 2.13-2.85, $2.46 \mathrm{~mm}$; total length/ wing length 1.95-2.38, 2.16; wing length / length of profemur 1.98-2.33, 2.12 .

Coloration. Head, thorax and abdomen brown, legs yellowish except distal fore femur and tarsus I light brown.

Head. Frontal tubercle absent. AR 3.07-3.69, 3.25. Temporal setae 15-25, 20; Clypeus with 20-33, 26 setae;

Palpomere lengths (in $\mu \mathrm{m}$ ): 38-55; 47; 115-153, 128; 123-163,141; 170-245, 208. Length of $5^{\text {th }}$ palpomere $/ 3^{\text {rd }}$ palpomere $1.42-2.04,1.61$.

Wing (Fig. 2A) VR 1.07-1.14, 1.10; Brachiolum with 2-3 setae; R with 20-29, 25 setae; $R_{1}$ with $13-21,17$ setae; $R_{4+5}$ with $12-18,15$ setae; squama with 9-19, 15 setae. Anal lobe developed.

Thorax. Dorsocentrals 8-13, 12; acorstichals 20-28, 23; prealars 5-7, 6; scutellum 10-18, 12 setae. 


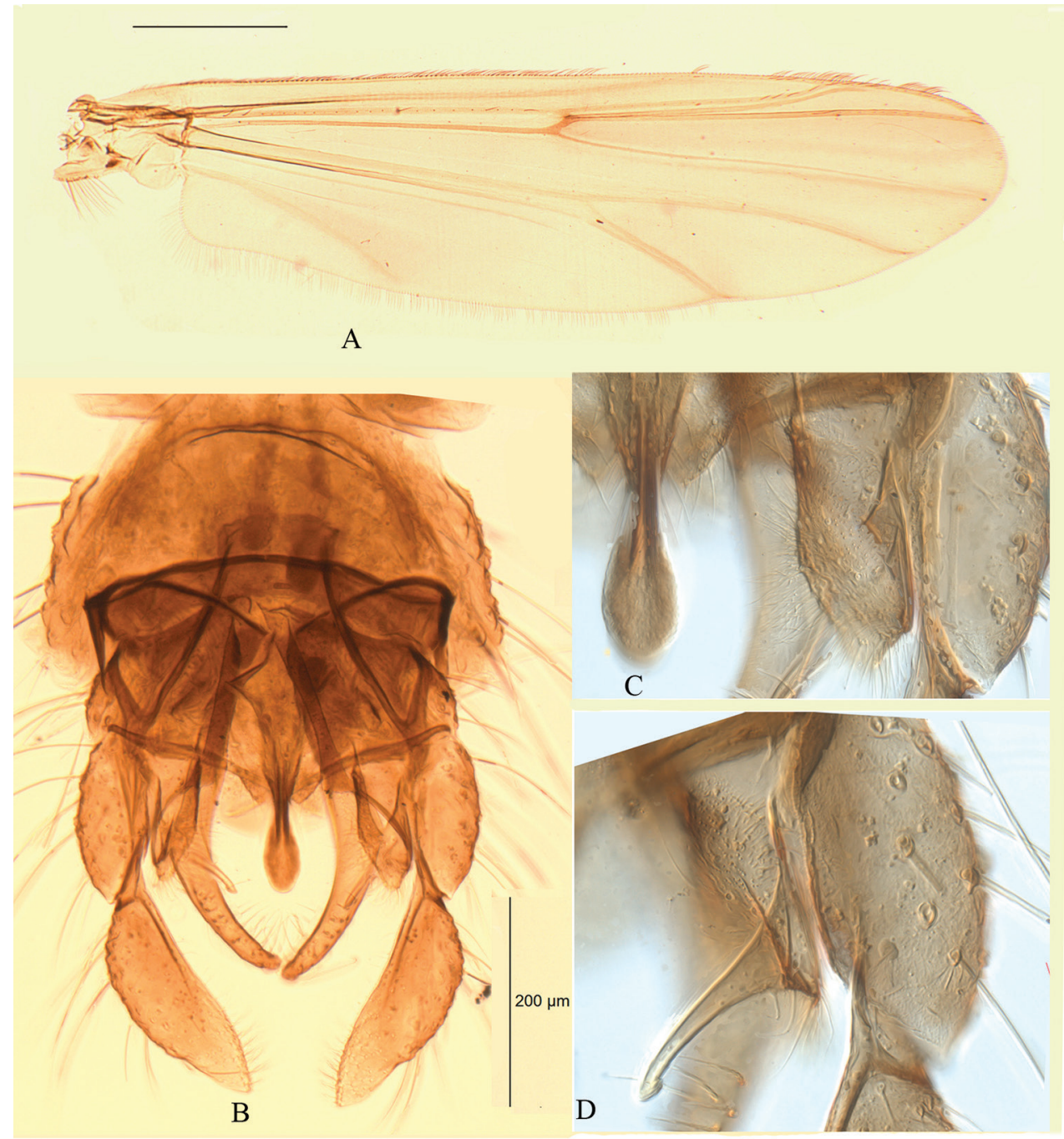

Figure 2. Kiefferulus trigonum sp. nov. male $\mathbf{A}$ wing $\mathbf{B}$ hypopygium in dorsal view $\mathbf{C}$ anal point and base of superior volsella $\mathbf{D}$ projection of superior volsella.

Legs. Tarsomere 1 of Mid and hind leg with 9-20, 14 and 6-17, 10 sensilla chaetica respectively. Front scale bluntly rounded; Spurs of mid tibia $23-38,29 \mu \mathrm{m}$, and 25-33, $29 \mu \mathrm{m}$ long, of hind tibia $28-38,32 \mu \mathrm{m}$, and 25-35, $29 \mu \mathrm{m}$. Width at apex of front tibia 75-98, $85 \mu \mathrm{m}$, of mid tibia 83-95, $89 \mu \mathrm{m}$, of hind tibia 90-110, $98 \mu \mathrm{m}$. Lengths and proportions of legs as the Table 1 .

Hypopygium (Figs 2B-D, 3). Anal tergite bands medially fused, and median anal tergite seta absent. Anal point basically narrow and apically broaden. Superior volsella with pad-like microchichiose and setose base, with long finger like projection inward to the apex of anal point. Inferior volsella slender, with strong distal setae. Gonocoxite 270-310, $293 \mu \mathrm{m}$ long, gonostylus 180-210, $198 \mu \mathrm{m}$, with numerous 
Table I. Length (in $\mu \mathrm{m}$ ) and proportions of legs of Kiefferulus trigonum sp. nov.

\begin{tabular}{lcccccc}
\hline & $\mathbf{f e}$ & $\mathbf{t i}$ & $\mathbf{t a}_{\mathbf{1}}$ & $\mathbf{t a}_{\mathbf{2}}$ & $\mathbf{t a}_{\mathbf{3}}$ & $\mathbf{t a}_{4}$ \\
\hline $\mathrm{P}_{1}$ & $1050-1325,1161$ & $825-1050,919$ & $1150-1500$, & $720-860,779$ & $630-800,712$ & $540-690,611$ \\
& & & 1322 & & & \\
$\mathrm{p}_{2}$ & $950-1250,1078$ & $820-1100,956$ & $520-650,582$ & $300-380,337$ & $230-300,263$ & $160-200,180$ \\
$\mathrm{p}_{3}$ & $1075-1350,1203$ & $1050-1375,1194$ & $470-940,777$ & $420-540,472$ & $360-480,418$ & $220-280,251$ \\
\hline & $\mathbf{t a}_{5}$ & $\mathbf{L R}$ & $\mathbf{B V}$ & $\mathbf{S V}$ & $\mathbf{B R}$ & \\
\hline $\mathrm{P}_{1}$ & $230-300,262$ & $1.38-1.52,1.44$ & $1.37-1.49,1.44$ & $1.53-1.63,1.57$ & $3.67-6.89,5.44$ & \\
$\mathrm{P}_{2}$ & $110-130,119$ & $0.58-0.65,0.61$ & $2.80-3.06,2.93$ & $3.32-3.67,3.49$ & $2.32-4.86,3.53$ & \\
$\mathrm{P}_{3}$ & $140-170,154$ & $0.66-0.69,0.68$ & $2.35-2.58,2.47$ & $2.87-3.10,2.97$ & $3.24-6.50,4.09$ & \\
\hline
\end{tabular}
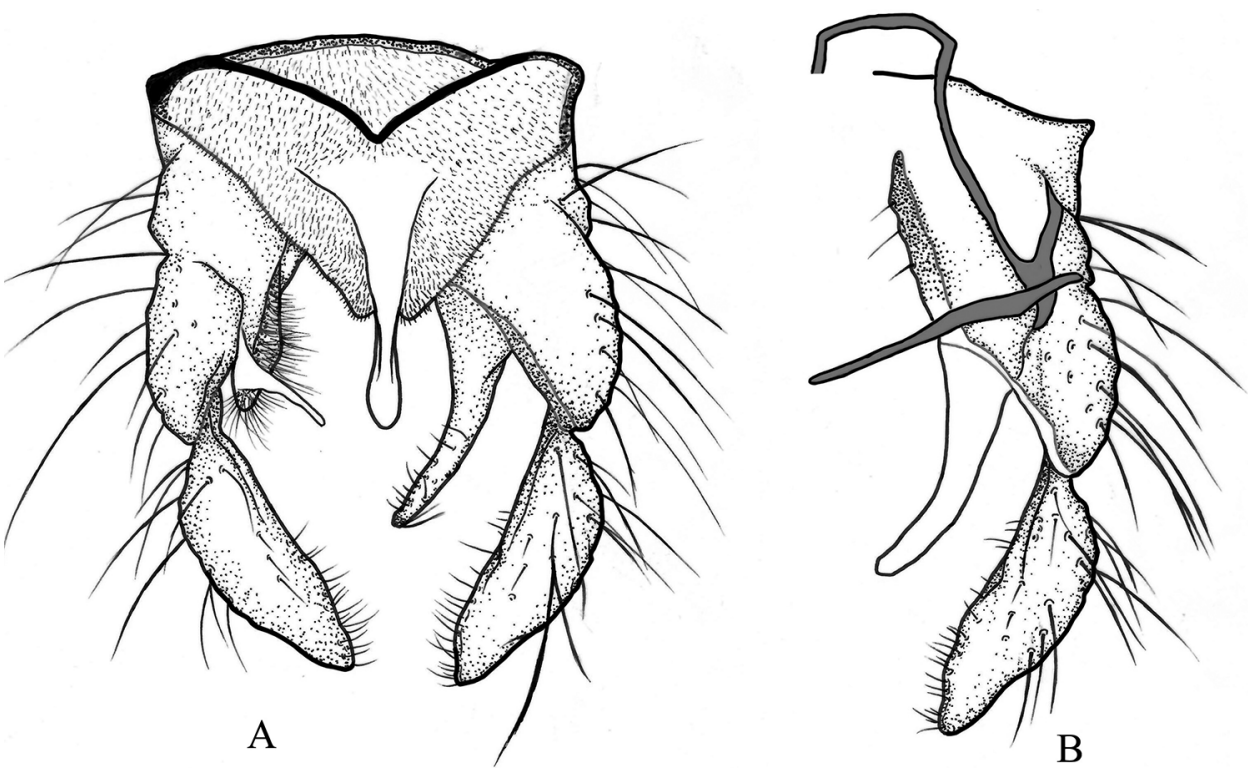

Figure 3. Kiefferulus trigonum sp. nov. male $\mathbf{A}$ hypopygium in dorsal view $\mathbf{B}$ hypopygium in ventral view.

distal microtrichia. Phallapodeme 220-250, $232 \mu \mathrm{m}$; transverse sternapodeme 100113, $108 \mu \mathrm{m}$. HR 1.42-1.61, 1.49; HV 1.95-2.88, 2.59.

Larva and female unknown.

Distribution. Fujian Province, Guizhou Province, Guangxi Zhuang Autonomous Region, and Hainan Province (Oriental China).

\section{Remarks}

Morphological characters such as the anal point narrow basally, distally broad, the superior volsella with microtrichia, and the gonostylus distally constricted positively and molecular phylogeny provide clues indicating the genus Kiefferulus. Morphologically, the new species shows great similarity with Einfeldia species with pad-like microtrichose and setose bases and a finger-like projection inwards to the apex of the anal point that clearly distinguishes them from species of Kiefferulus. 


\section{Acknowledgements}

This paper forms part of the project "Phylogeny of the subfamily Chironominae". The first author is indebted to Elisabeth Stur and Torbjørn Ekrem (NTNU University Museum, Trondheim, Norway) when studying in Trondheim. Many thanks to Yu Xue, who did a lot of work on Kiefferulus and Mingxuan Hong for figures drawing in preparing the manuscript. Financial support is acknowledged from Zhejiang Provincial Natural Science Foundation of China (LY17C040001) and the National Natural Science Foundation of China (31672264).

\section{References}

Andersen T, Cranston PS, Epler JH (2013) The larvae of Chironomidae (Diptera) of the Holarctic Region - Keys and diagnoses. Insect Systematics \& Evolution Supplement 66:1-571.

Andersen T, Mendes HF, Pinho LC (2017) Two new Neotropical Chironominae genera (Diptera: Chironomidae). CHIRONOMUS Journal of Chironomidae Research 30: 26-54. https://doi.org/10.5324/cjcr.v0i30.2029

Castresana J (2000) Selection of conserved blocks from multiple alignments for their use in phylogenetic analysis. Molecular Biology and Evolution 17(4): 540-552. https://doi. org/10.1093/oxfordjournals.molbev.a026334

Cranston PS, Dillon ME, Pinder LCV, Reiss F (1989) The adult males of Chironominae (Diptera, Chironomidae) of the Holarctic region. Keys and diagnoses. In: Wiederholm T (Ed.) The adult males of Chironomidae (Diptera) of the Holarctic region - Keys and diagnoses. Entomologica scandinavica Supplement 34: 353-502.

Cranston PS, Hardy NB, Morse GE (2012) A dated molecular phylogeny for the Chironomidae (Diptera). Systematic Entomology 37(1): 172-188. https://doi.org/10.1111/j.13653113.2011.00603.x

Cranston PS, Martin J, Mulder M, Spies M (2016) Clarification of Einfeldia Kieffer, 1922 (Diptera: Chironomidae) with E. australiensis (Freeman, 1961), comb. n. based on immature stages. Zootaxa 4158(4): 491-506. https://doi.org/10.11646/zootaxa.4158.4.3

Dereeper A, Guignon V, Blanc G, Audic S, Buffet S, Chevenet F, Dufayard JF, Guindon S, Lefort V, Lescot M, Claverie JM, Gascuel O (2008) Phylogeny.fr: robust phylogenetic analysis for the non-specialist. Nucleic Acids Research 36 (Web Server issue): W465-469. https://doi.org/10.1093/nar/gkn180

Edgar RC (2004) MUSCLE: multiple sequence alignment with high accuracy and high throughput. Nucleic Acids Research 32(5): 1792-1797. https://doi.org/10.1093/nar/gkh340

Folmer O, Black M, Hoeh W, Lutz R, Vrijenhoek R (1994) DNA primers for amplification of mitochondrial cytochrome c oxidase subunit I from diverse metazoan invertebrates. Molecular Marine Biology and Biotechnology 3(5): 294-299.

Garnett ST, Christidis L (2017) Taxonomy anarchy hampers conservation Taxonomy anarchy hampers conservation. Nature 546(7656): 25-27. https://doi.org/10.1038/546025a

Hall TA (1999) BioEdit: a user-friendly biological sequence alignment editor and analysis program for Windows 95/98/NT. Nucleic acids symposium series 41: 95-98. 
Hamilton AL, Saether OA, Oliver DR (1969) A Classification of the Nearctic Chironomidae. Fisheries research board of canada technical report 124: 1-42.

Holstein N, Luebert F (2017) Taxonomy: stable taxon boundaries. Nature 548(7666): 158158. https://doi.org/10.1038/548158d

Kohshima S (1984) A novel cold-tolerant insect found in a Himalayan glacier. Nature 310(5974): 225-227. https://doi.org/10.1038/310225a0

Kumar S, Stecher G, Tamura K (2016) MEGA7: Molecular Evolutionary Genetics Analysis Version 7.0 for Bigger Datasets. Molecular Biology and Evolution 33(7): 1870-1874.

Lanfear R, Calcott B, Ho SYW, Guindon S (2012) PartitionFinder: combined selection of partitioning schemes and substitution models for phylogenetic analyses. Molecular Biology and Evolution 29(6): 1695-1701.

Linnaeus C (1753) Species plantarum (Vol. 2). Stockholm: Laurentius Salvius.

Linnevich AA (1971) The Chironomidae of Lake Baikal. Limnologica (Berlin) 8: 51-52.

Nylander JAA, Ronquist F, Huelsenbeck JP, Nieves-Aldrey JL (2004) Bayesian phylogenetic analysis of combined data. Systematic Biology 53(1): 47-67. https://doi. org/10.1080/10635150490264699

Padial JM, Miralles A, De la Riva I, Vences M (2010) The integrative future of taxonomy. Frontiers in Zoology 7: 16. https://doi.org/10.1186/1742-9994-7-16

Qi X, Lin XL, Ekrem T, Beutel RG, Song C, Orlov I, Chen CT, Wang XH (2019) A new surface gliding species of Chironomidae: An independent invasion of marine environments and its evolutionary implications. Zoologica Scripta 48(1): 81-92.

Rambaut A, Suchard MA, Xie D, Drummond AJ (2014) Tracerv1.6. http://beast.bio.ed.ac.uk/ Tracer.2014

Ratnasingham S, Hebert PDN (2007) BOLD: The Barcode of Life Data System (www.barcodinglife.org). Molecular Ecology Notes 7(3): 355-364. https://doi.org/10.1111/j.14718286.2007.01678.x

Sæther OA (1969) Some Nearctic Pondonominae, Diamesinae, and Orthocladiinae (Diptera: Chironomidae). Bulletin of the Fisheries Research Board of Canada 170: 1-154.

Sæther OA (1980) Glossary of chironomid morphology terminology (Diptera: Chironomidae). Entomologica scandinavica Supplement 14: 1-51.

Scotland RW, Olmstead RG, Bennett JR (2003) Phylogeny reconstruction: the role of morphology. Systematic Biology 52(4): 539-548. https://doi.org/10.1080/10635150309309

Silvestro D, Michalak I (2012) raxmlGUI: a graphical front-end for RAxML. Organisms Diversity \& Evolution 12(4): 335-337. https://doi.org/10.1007/s13127-011-0056-0

Thomson SA, Pyle RL, Ahyong ST, Alonso-Zarazaga M, Ammirati J, Araya JF, et al. (2018) Taxonomy based on science is necessary for global conservation. Plos Biology 16(3).

Townes HK (1945) The Nearctic Species of Tendipedini - Diptera, Tendipedidae (= Chironomidae). American Midland Naturalist 34(1): 1-206. https://doi.org/10.2307/2421112

Wiederholm T (1986) The pupae of Chironomidae of the Holarctic region - Keys and diagnoses. Entomologica scandinavica Supplement 28: 1-482.

Wiederholm T (1989) The adult males of Chironomidae of the Holarctic region - Keys and diagnoses. Entomologica scandinavica Supplement 34:1-532. 


\section{Supplementary material I}

The concatenated DNA dataset used for phylogenetic analyses.

Authors: Chao Song, Xinhua Wang, Wenjun Bu, Xin Qi

Data type: NEX file

Copyright notice: This dataset is made available under the Open Database License (http://opendatacommons.org/licenses/odbl/1.0/). The Open Database License $(\mathrm{ODbL})$ is a license agreement intended to allow users to freely share, modify, and use this Dataset while maintaining this same freedom for others, provided that the original source and author(s) are credited.

Link: https://doi.org/10.3897/zookeys.909.39347.suppl1 